\title{
NUMERICAL SIMULATION OF WOOD-POLYMER COMPOSITES EXTRUSION
}

\author{
Fabian Liese $^{1}$, Olaf Wünsch ${ }^{1}$, Serhiy Yatsenko ${ }^{2}$, Johannes Rudloff ${ }^{2}$, Marieluise Lang ${ }^{2}$, \\ Thomas Hochrein $^{2}$ and Martin Bastian ${ }^{2}$ \\ ${ }^{1}$ University of Kassel \\ D-34125 Kassel \\ fluiddynamics@uni-kassel.de and www.uni-kassel.de \\ ${ }^{2}$ SKZ-KFE gGmbH \\ D-97076 Würzburg \\ s.yatsenko@skz.de and www.skz.de
}

Key words: WPC, non-Newtonian, extrusion, rheology, CFD

\begin{abstract}
The combination of fillers and polymers is used to influence the mechanical, optical and processing properties of the base material and is finding more and more applications. The addition of wood to polymers results in wood-polymer composites (WPC), whereby the wood content can be up to $80 \mathrm{wt}$ \%. This composite is mainly processed to deckings by means of extrusion. The extrusion die is an essential part of the processing procedure, which determines both process parameters and the final product. A special feature of WPC extrusion dies is cooling plate, which leads to partial solidification of the melt before leaving the die. This solidification is necessary to ensure dimensionally stable extrusion without strand breakage.

For the design of WPC extrusion dies, numerical simulations are increasingly used in addition to empirical data, whereby OpenFOAM is used in the present investigations. Based on rheological measurements, both the shear thinning flow behavior and the temperature dependence of the viscosity are modeled. The partial solidification of the melt at the cooling plate before the outlet is modeled in a single-phase over a step in viscosity. In addition, the solidified melt slips on the cooling wall, which is also taken into account in the model.

In the parallel zone, there is a pure shear flow, while in the transition elements such as the flange to the extruder or at the mandrel, uni- and biaxial extension components are dominant due to the crosssectional changes. High-density polyethylen (HDPE) with wood flour exibits a shear thinning and extensional thickening behavior, so that different models are necessary for description. To calculate the stresses, interpolation is performed between the different models with respect to the invariants according to Böhme [1], depending on the type of flow present. To validate the numerical simulations, an extrusion die for a square hollow profile with pressure and temperature sensors along the flow direction is subject to experimental measurements. The aim of the numerical simulations is to investigate the flow in more detail and to give optimization hints.
\end{abstract}


Liese, F. et al.

\section{INTRODUCTION}

The market share of wood-polymer composites has more than doubled in the building and automotive industries since 2016 [2], with extrusion into deckings on terraces, balconies, etc. taking the main part. Through WPC, the positive properties of wood appearance are combined with the weather resistance of polymers. While the use of computational fluid dynamics (CFD) is now widespread in the design of extrusion dies for standard polymers, the processing of wood-polymer composites usually relies on empirical knowledge. This is mainly due to the complex thermorheological material behavior as well as wall slip and solidification effects, which have to be considered in the flow simulation [3].

The rheological behavior of WPC with different wood contents was studied by Wolcott [4] and the wall slip of polymer melts was investigated by Rosenbaum [5]. The extrusion of polymer melts in terms of pressure drop and velocity variance at the outlet was also studied $[6,7,9]$. However, a comparison of numerical with experimental data for the pressure at different positions during the extrusion process of WPC with the combination of shear and extensional viscosity is missing so far.

In the context of this work, thermorheological data is measured and modeled with different material models. Numerical simulations are used to investigate the flow behavior during the extrusion process on an experimental die with a square hollow profile in more detail, and the pressure variation with different material models is compared with experimentally determined data provided by German Plastics Center (SKZ) [8].

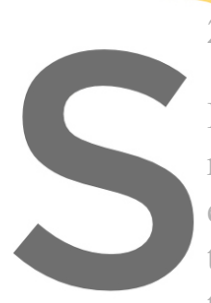

2 Modeling

In order to determine the thermorheological behavior of the melt,
rotational and high-pressure capillary rheometer. These measu remen
eling. In addition to the shear viscosity $\eta$, the extensional viscosity
temperature is also of relevance. The extrusion temperatures of WP

that the material characteristics are also determined in this range.

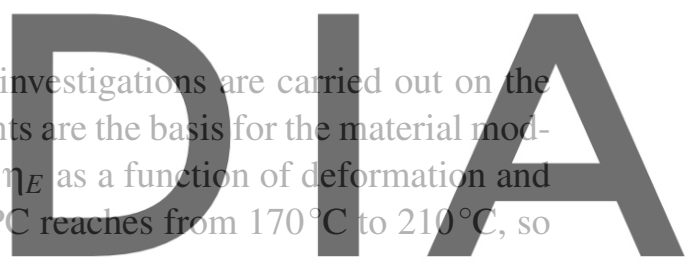

Figure 1 shows the shear viscosity as a function of shear rate of HPPE with. a weight wood fraction of Register for frege at htt

to $10^{-1} \mathrm{~s}^{-1}$ are collected using a rotational rheometer with a plate-plate system. To suppress oxidation,

the measurement chamber is fiooded with nitrogen. For larger shear rates starting at $10^{0} \mathrm{~s}^{-1}$, a capiliary rheometer is used. A shear thinning behavior is formed with a first Newtonian plateau, which disappears with increasing wood content. This suggests the formation of a yield point, which can also be confirmed in separate shear stress controlled measurements and is consistent with the measurements from Wolcott [4]. The zero viscosity $\eta_{0}$ at $30 \%$ wood content is about $10^{5}$ Pas.

Figure 2 shows both the shear viscosity $\eta$ as a function of shear rate $\dot{\gamma}$ and the extensional viscosity $\eta_{E}$ as a function of the extension rate $\dot{\varepsilon}$ of HDPE with $30 \%$ wood content. For the rest of the work, WPC with $30 \%$ is investigated in more detail. Measurements are recorded at temperatures ranging from $170^{\circ} \mathrm{C}$ to $210^{\circ} \mathrm{C}$. The extensional viscosity corresponds to three times the shear viscosity for small deformations. With increasing deformation, both shear thinning and extensional thickening behavior is evident such that the trouton ratio increases with rising deformation. Measurement data could be carried out up to about $6 \cdot 10^{-1} \mathrm{~s}^{-1}$ with the rheotens setup of the capillary rheometer, after which the strand breaks off. With increasing temperature, both shear and extensional viscosity decrease. 


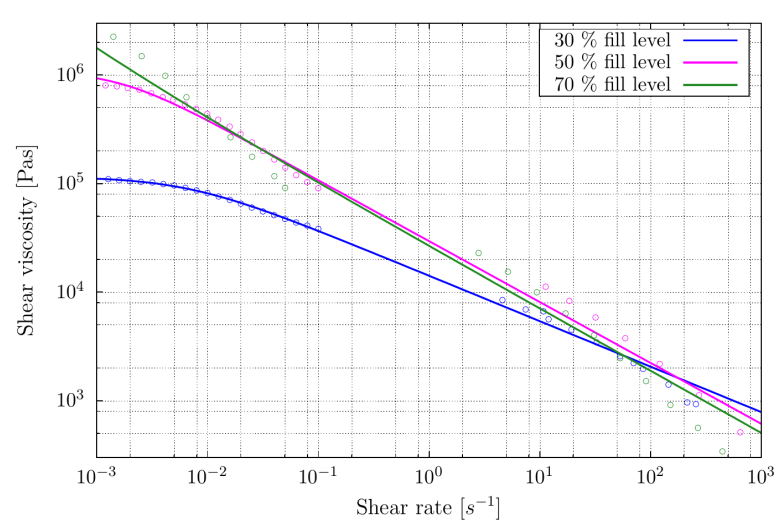

Figure 1: Influence of wood content at $30 \%$ to $70 \%$ on Figure 2: Shear and extensional viscosity of $30 \%$ WPC the shear viscosity as a function of shear rate at $190^{\circ} \mathrm{C}$. at different temperatures as a function of deformation.

A Carreau-Yasuda model [10] is used to model the shear thinning behavior, which is shown in equation (1a). The determination of the model parameters is performed at the temperature $T_{\text {ref }}=190^{\circ} \mathrm{C}$. In this model, in addition to the dependence on shear rate, the dependence of zero viscosity on temperature is also modeled via a shift function $a_{T}$, which is necessary in an extrusion process. The extensional behavior is also modeled via a Carreau-Yasuda model according to equation (1b), where the flow index $n_{E}$ is greater than one. For numerical stabilization, the extensional viscosity is limited to a value of

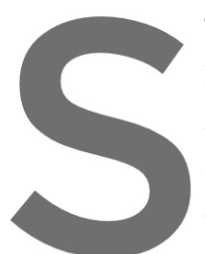
$\eta_{E, l i m}=10^{7}$ Pas. Howeve not a limitation.

According to equation approach [11] and for tem describes the transition to of the melt is modeled
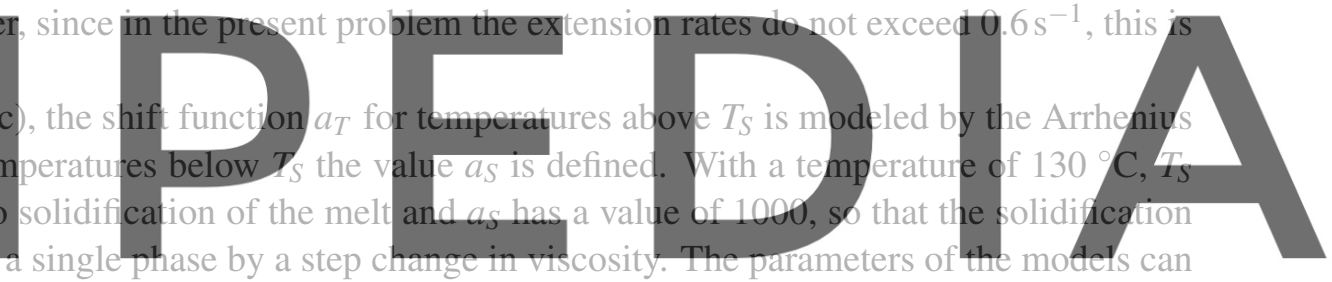

be taken from table 1 .

Register for free at https//www.scipedia.com to download the version without the watermark

$$
\begin{aligned}
\eta(\dot{\gamma}, T) & =a_{T} \cdot \eta\left(\dot{\gamma}, T_{r e f}\right)=a_{T} \cdot \eta_{0}\left[1+\left(\lambda_{S}|\dot{\gamma}|\right)^{\alpha}\right]^{\frac{n-1}{\alpha}} \\
\eta_{E}(\dot{\varepsilon}, T) & =a_{T} \cdot \eta_{E}\left(\dot{\varepsilon}, T_{r e f}\right)=a_{T} \cdot \eta_{0, E}\left[1+\left(\lambda_{E}|\dot{\varepsilon}|\right)^{\alpha_{E}}\right]^{\frac{n_{E}-1}{\alpha_{E}}} \\
a_{T} & = \begin{cases}\exp \left[\frac{E_{0}}{R_{0}}\left(\frac{1}{T}-\frac{1}{T_{0}}\right)\right] & T>T_{S} \\
a_{S} & T \leq T_{S}\end{cases}
\end{aligned}
$$

In the equations (2) the invariants of the strain-rate tensor $\mathbf{D}$ for an incompressible flow are defined [13]. From the velocity gradient, the strain-rate tensor is defined as $\mathbf{D}=\frac{1}{2}\left(\operatorname{grad} \mathbf{v}+(\operatorname{grad} \mathbf{v})^{T}\right)$. Due to incompressibility, the first invariant is zero and the second invariant simplifies to an always negative expression. The third invariant corresponds to the determinant of $\mathbf{D}$ and can take both positive and negative values. From the second invariant, a representative shear rate for three-dimensional flows is given by equation (3a). The extension rate $\dot{\varepsilon}$ in equation (3b) presented here is valid for an uniaxial extension flow [1]. 
This formulation is chosen since an uniaxial extension state is present in the measurements of the extensional viscosity with the rheotens setup of the capillary rheometer. To further classify the deformation state, the parameter $\kappa$ is introduced in equation (3c), which is the ratio of third to second invariant [14]. When $\kappa=0$, a pure shear flow is present. At the positive limit of the invariant space of 0.385 , the fluid is strained uniaxial and at the negative limit of -0.385 , it is strained biaxial. In between, there are mixed deformation states and no flow state is possible outside the mentioned limit values.

$$
\begin{aligned}
I_{D} & =\operatorname{tr}(\mathbf{D})=0 \\
I I_{D} & =-\frac{1}{2} \operatorname{tr}\left(\mathbf{D}^{2}\right)<0 \\
I I I_{D} & =\operatorname{det}(\mathbf{D})
\end{aligned}
$$$$
\dot{\gamma}=\sqrt{-4 I I_{D}}
$$$$
\dot{\varepsilon}=\frac{-3 I I I_{D}}{I I_{D}}
$$$$
\kappa=\frac{I I I_{D}}{\left(-I I_{D}\right)^{\frac{3}{2}}}
$$

Viscosity models for pure shear (1a) and extensional viscosity (1b) as well as representative deformation quantities (3) are defined. To account for both shear and extensional viscosity when calculating the viscous stress tensor $\mathbf{T}=\phi_{1} \mathbf{D}$ in a generalized Newtonian material model, a hybrid model [1] is used for the functional $\phi_{1}$.
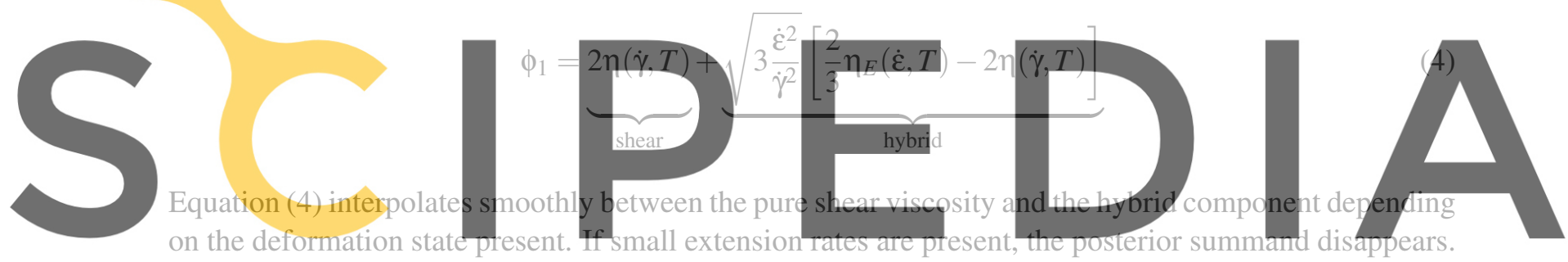

However, if the extension rate is large in relation to the shear rate, the hybrid component with the exten-

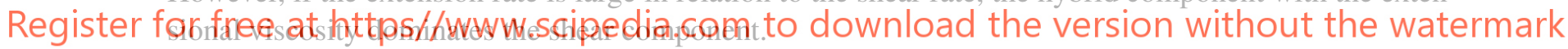

For the numerical solution of the flow problem, the mass balance (5) for incompressible fluids and the momentum equation (6) are solved neglecting volume forces.

$$
\begin{gathered}
\operatorname{div} \mathbf{v}=0 \\
\rho \frac{D \mathbf{v}}{D t}=-\operatorname{grad} p+\operatorname{div} \mathbf{T}
\end{gathered}
$$

In order to analyze the thermal variation during the extrusion process, the energy equation (7) is solved.

$$
\rho c_{p} \frac{D T}{D t}=\operatorname{div}(\lambda \operatorname{grad} T)+\operatorname{tr}(\mathbf{T} \cdot \mathbf{D})+\rho \dot{q}
$$

Due to the high viscosities and shear rates especially in the parallel zone of the extrusion die, the viscous friction is considered via the term $\operatorname{tr}(\mathbf{T} \cdot \mathbf{D})$ [16]. In addition, the source term $\rho \dot{q}$ is solved to calculate 
the enthalpy during solidification. The variation of the specific heat flux $\dot{q}(T)$ is acquired by differential scanning calorimetry (DSC) measurements at a cooling rate of $10 \mathrm{Kmin}^{-1}$ and modeled by a modified Ziabicki model $[12,15]$.

Table 1: Material parameters of HDPE with $30 \%$ wood content.

\begin{tabular}{lll|lll|lll|lll}
\hline$\eta_{0}$ & {$[\mathrm{Pas}]$} & 115515 & $\eta_{0, E}$ & {$[\mathrm{Pas}]$} & 346545 & $E_{0}$ & {$\left[\mathrm{~J} \mathrm{~mol}^{-1}\right]$} & 24086 & $\rho$ & {$\left[\mathrm{kg} \mathrm{m}^{-3}\right]$} & 850 \\
\hline$\lambda_{S}$ & {$[\mathrm{~s}]$} & 149.69 & $\lambda_{E}$ & {$[\mathrm{~s}]$} & 10.42 & $R_{0}$ & {$\left[\mathrm{~J} \mathrm{~K}^{-1} \mathrm{~mol}^{-1}\right]$} & 8.314 & $c_{p}$ & {$\left[\mathrm{~J} \mathrm{~kg}^{-1} \mathrm{~K}^{-1}\right]$} & 2580 \\
\hline$\alpha$ & {$[-]$} & 1.128 & $\alpha_{E}$ & {$[-]$} & 1.011 & $T_{0}$ & {$[\mathrm{~K}]$} & 396.95 & $\lambda$ & {$\left[\mathrm{W} \mathrm{m}^{-1} \mathrm{~K}^{-1}\right]$} & 0.3158 \\
\hline$n$ & {$[-]$} & 0.581 & $n_{E}$ & {$[-]$} & 1.959 & $T_{S}$ & {$[\mathrm{~K}]$} & 403.05 & & & \\
\cline { 1 - 5 }
\end{tabular}

The remaining material parameters such as density $\rho$, specific heat capacity $c_{p}$ and thermal conductivity $\lambda$ are assumed to be constant and can be taken from table 1 .

\section{Numerical Simulations}

By the use of numerical simulations in OpenFOAM, the flow behavior in the entire extrusion die can be investigated and a design of the relevant components is possible without time-consuming and costintensive manufacturing. By comparing the numerically simulated pressure drop at different locations with experimental data provided by SKZ, the quality of the numerical model can be evaluated.
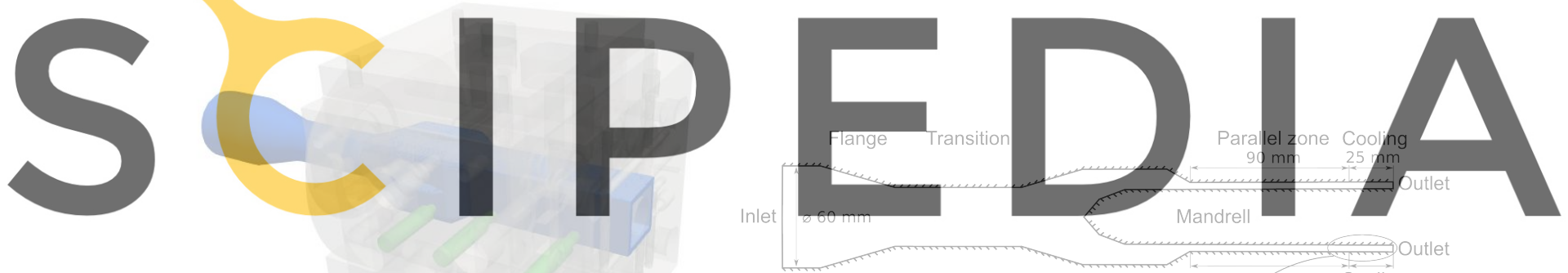

Register for free at https//www.scipedia.com to download the version without thelivatermark

Figure 3: Extrusion die with flow area in blue and pressure sensors in green.
Figure 4: Slice of flow area and mesh of parallel zone in detail view.

In Figure 3, the entire extrusion die with all tool, heating and cooling plates is shown opaque. In blue the flow area and in green pressure sensors are shown, which measure the pressure at the surface of the die. The flow area is meshed from the circular connection to a counter-rotating twin screw extruder at the inlet to the outlet at the parallel zone of a square hollow profile.

In Figure 4, the dimensioned 2D cross-section of the 3D flow area with the various components along the die is sketched. Since partial solidification within the mold is necessary for dimensionally stable extrusion of WPC, a $25 \mathrm{~mm}$ wide cooling plate is placed in front of the outlet. The mesh is exemplified in the detail view of the parallel zone, with 20 hexahedra discretizing the $4 \mathrm{~mm}$ height of the channel. 
Liese, F. et al.

\subsection{Boundary Conditions}

In order to compare the pressure between experiment and numerics, the mass flow at the inlet is varied between $20 \mathrm{~kg} \mathrm{~h}^{-1}$ to $40 \mathrm{~kg} \mathrm{~h}^{-1}$. The temperature is set to $190^{\circ} \mathrm{C}$ at all walls and at the inlet, only at the cooling plate a temperature of $60^{\circ} \mathrm{C}$ is specified. This corresponds to standard temperatures during the extrusion process of WPC and is measured by temperature sensors in the die during the experiments.

The WPC melt partially solidifies on the surface of the cooling plate. In the experiments, it can be seen that the solidified profile slips out of the die. To account this slipping along the cooling wall, the mean flow velocity in the parallel zone $u_{b u l k}$ is set as a boundary condition in the extrusion direction.

From the material parameters in table 1 and the boundary conditions in the parallel zone, dimensionless numbers can be determined to evaluate the flow at $30 \mathrm{~kg} \mathrm{~h}^{-1}$. Based on the very small Reynolds number of $\operatorname{Re}=\rho u_{B u l k} d_{h} / \eta_{0}=2.63 \cdot 10^{-6}$, it can be argued that a creeping flow is present. The Prandtl number with $\operatorname{Pr}=c_{p} \eta_{0} / \lambda=9.44 \cdot 10^{8}$ indicates a small temperature boundary layer and from the Péclet number $\mathrm{Pe}=\operatorname{Re} \cdot \mathrm{Pr}=2483$ it is clear that convective heat transport is dominant over diffusive heat transport.

\subsection{Results}

To evaluate the numerical simulation, the flow variables are first analyzed on the symmetry plane and the pressure distribution along the die is compared with the experimental data at four measuring points. In Figure 5 the magnitude of the velocity along the extrusion die is shown. The flow is symmetric and the fluid accelerates in front of the mandrel as well as in the parallel zone due to cross-sectional contraction.

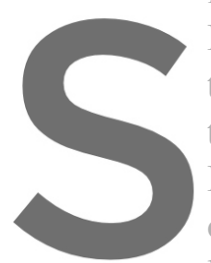
In the detailed view, the the cooling wall, an asymmetric to the outlet.

In figure 6 the temperature field of large shear, which is $\mathrm{m}$ low and there is almost no cross flow, the temperatur
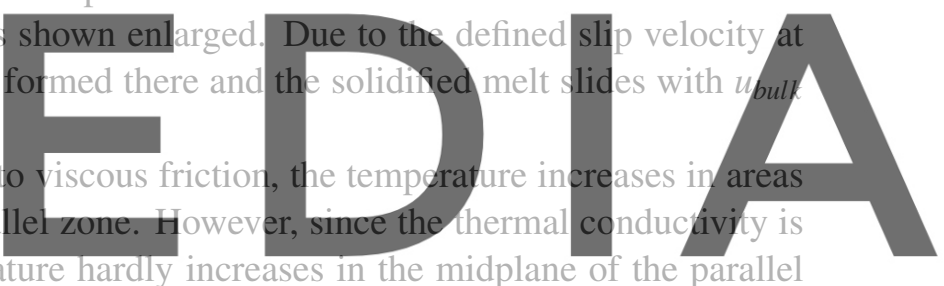

zone. The formation of the temperature boundary layer at the cooling wall.can be. clearly seen in the

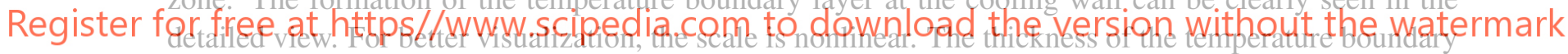

layer increases with the cooling wall length and is $20 \%$ of the channel height at the outlet.

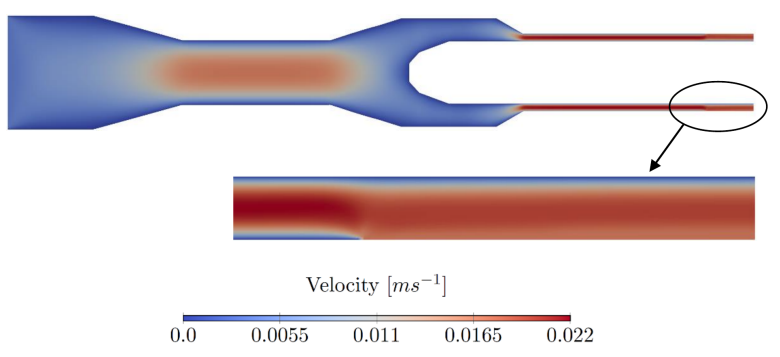

Figure 5: Velocity on symmetry plane.

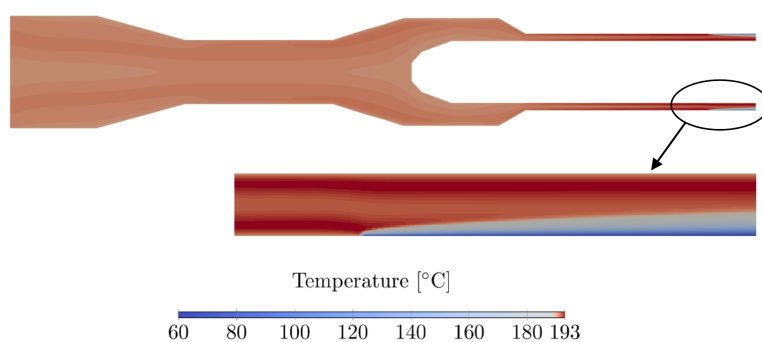

Figure 6: Temperature on symmetry plane.

To further evaluate the flow, Figure 7 shows deformation variables such as the shear rate, extension rate, as well as the parameter $\kappa$ along the extrusion die in the left column, and the associated viscosities in the right column. 
Liese, F. et al.

The shear rates are present in a wide range from $10^{-2} \mathrm{~s}^{-1}$ to $10^{2} \mathrm{~s}^{-1}$, with shear rates increasing toward the walls. Due to wall slip at the cooling plate before the outlet, the shear rate there is significantly reduced. The corresponding shear thinning viscosity in the right column increases with a decrease in shear rate to zero viscosity of $10^{5}$ Pas and decreases toward the walls with an increase in shear rate.

The extension rate according to equation (3b) in the second line has a maximum of $0.6 \mathrm{~s}^{-1}$. Comparing with Figure 2, it can be seen that this deformation range is covered by the measured data and extensional thickening is present. At locations with a change in cross-section, such as after the inlet and before the mandrel, the extension rate increases and on the other hand decreases towards zero for constant crosssection. Due to the extensional thickening flow behavior, the extensional viscosity also increases with a rising extension rate, which can be seen in the column to the right of the deformation. The extensional viscosity is overall 1-2 decades larger than the shear viscosity and has a maximum of $1.6 \cdot 10^{6}$ Pas.

Which type of deformation is present can be evaluated in Figure 7 bottom left by the $\kappa$-value according to equation (3c). In opaque displayed regions with value 0 , pure shear is present, for negative limit value of -0.385 biaxial extension and for positive limit value of 0.385 uniaxial extension is present. After the inlet, the fluid is uniaxially strained in the circular cross-sectional contraction. The mandrel divides the flow area from the circular cross-section into a square hollow section, so the fluid is strained not only uniaxially but also biaxially. Due to the solidification of the melt at the cooling wall, the flow crosssection contracts along the length of the cooling plate and the fluid is also strained at the transition. To account for both shear and extensional viscosity, the hybrid model given by equation (4) is used, with the resulting viscosity shown in the lower right of the figure 7. In regions of constant cross section, where pure shear is present, there is no difference from pure shear viscosity. However, the viscosity increases
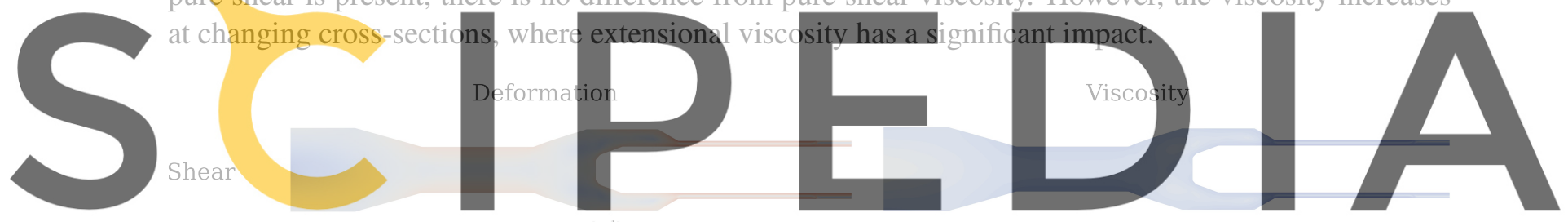

Register for free at https//WWW.scipedia.com to download the version without the watermark Extension
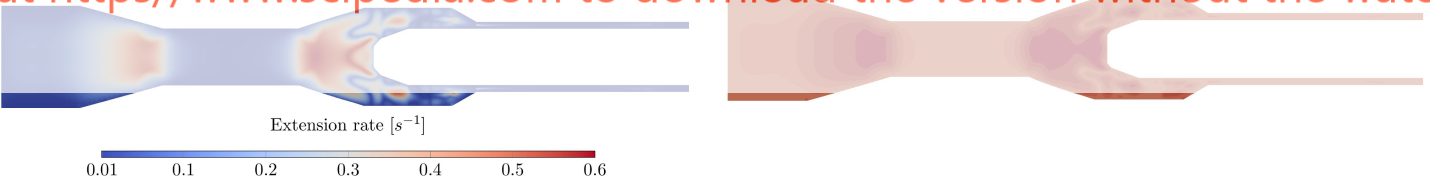

Hybrid
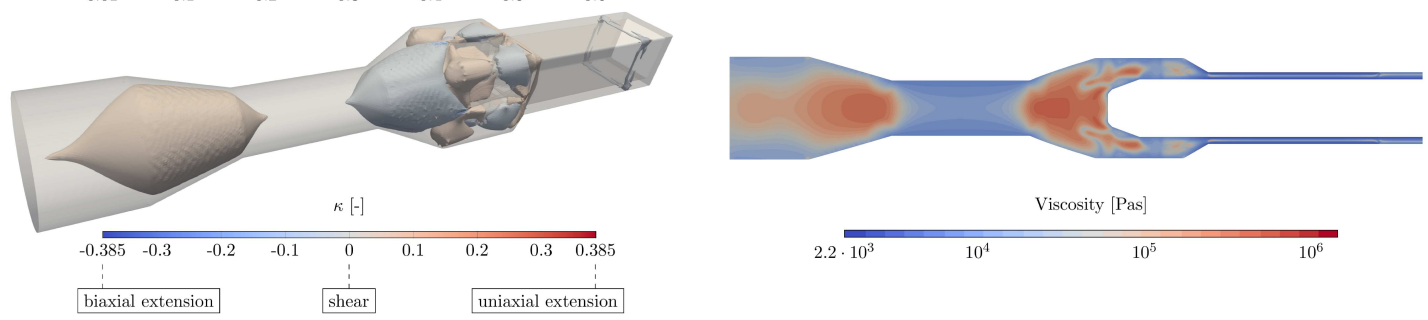

Figure 7: Deformations and viscosities along the extrusion die.

In order to investigate the influence of the different material models on the pressure drop and to perform a comparison with the experimental data at different mass flows, the pressure contour at the surface of 
the die of the hybrid material model with a mass flow of $30 \mathrm{~kg} \mathrm{~h}^{-1}$ is shown in the lower area in Figure 8 . In addition, the measuring points $s_{p 0}$ to $s_{p 3}$ are visualized in green, where at the point $s_{p 0}$ the pressure is measured by sword sensor and at the points $s_{p 1}$ to $s_{p 3}$ at the surface of the flow channel.

The diagram shows the pressure curve along the die length from both the hybrid material model according to equation (4) and with the pure shear viscosity model according to equation (1a). The measured data at locations $s_{p 0}$ to $s_{p 4}$ are also shown.

In the parallel zone, there is almost no difference between the shear and hybrid models in the numerical simulations due to the presence of the plane unidirectional flow. At the measuring points $s_{p 2}$ and $s_{p 3}$ in the parallel zone, the deviation between measurements and simulation is small.

In the experimental data however, there is a noticeable pressure drop between $s_{p 1}$ and $s_{p 2}$. This is not represented numerically solely with a shear viscosity model. This can be explained by the fact that the flow cross-section in front of the parallel zone is significantly larger than in the parallel zone itself, which is why a kink in the graph of the numerical pressure can be seen. In the hybrid model, this kink is much flatter due to the viscosity increase caused by the consideration of extensional thickening.

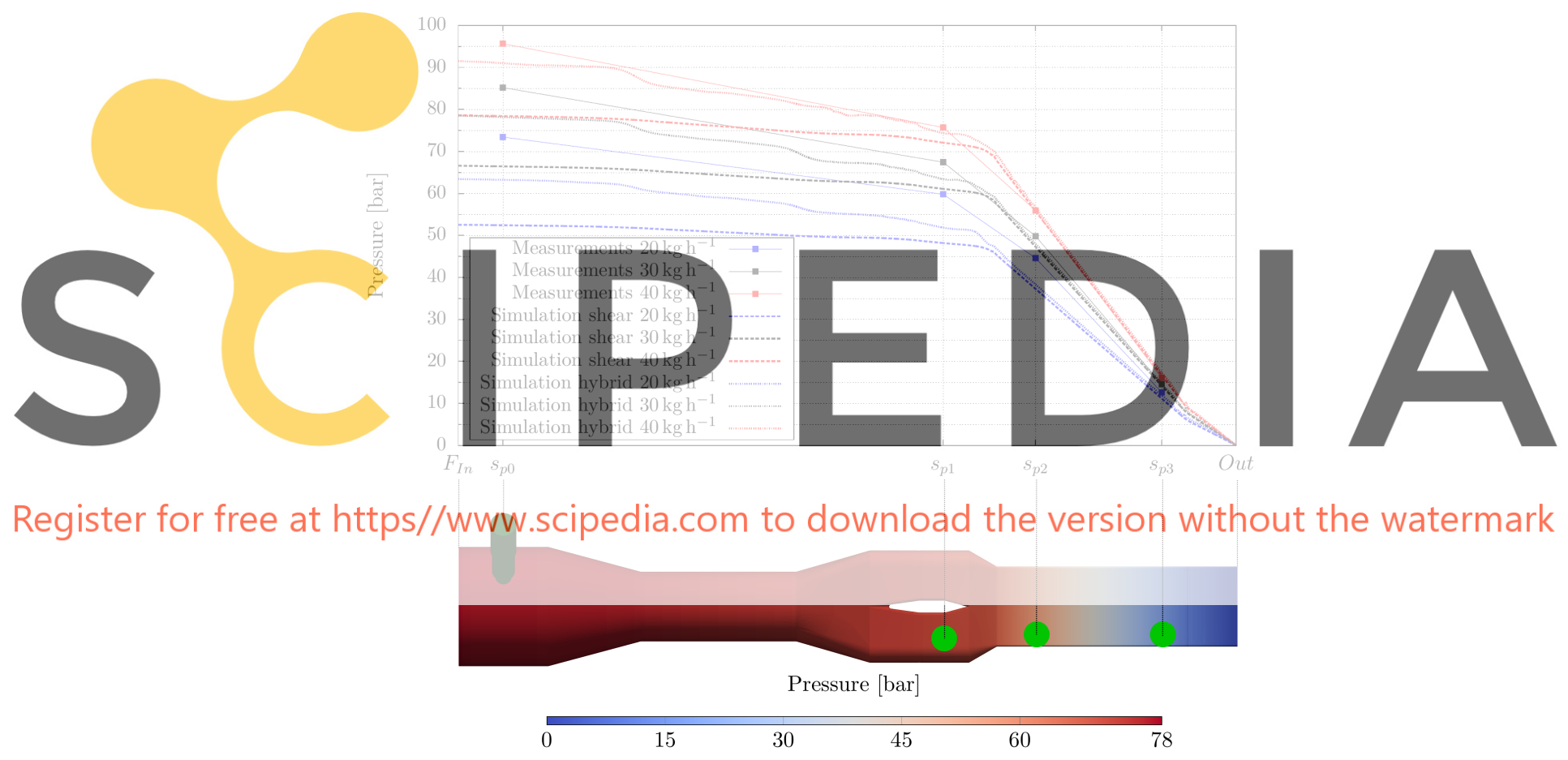

Figure 8: Pressure distribution from measurements and simulation.

Between $s_{p 0}$ and $s_{p 1}$, this phenomenon is even more pronounced. Experimentally, a pressure drop of about 15 bar results, while with the numerical model with the pure shear viscosity this pressure drop is below 5 bar over all mass flows. This also seems plausible due to the large flow cross-section in a creeping flow. However, to numerically represent the pressure increase observed experimentally between $s_{p 0}$ and $s_{p 1}$, the use of the hybrid material model is necessary. This increases the viscosity and thus the pressure loss in the presence of extension, which occurs with changes in cross-section. 


\section{CONCLUSIONS AND OUTLOOK}

In this work, the flow behavior of wood-polymer composites during the extrusion process is simulated and compared with experimental data. Rheological investigations are used to determine shear thinning and extensional thickening behavior. Solidification and wall slip effects are also considered in the simulation. In addition to the Carreau-Yasuda model in combination with an Arrhenius function for shear and extensional viscosity, a hybrid viscosity model is also used for the numerical simulations, which smoothly interpolates between shear and extensional viscosity depending on the deformation state present. When comparing the pressure between simulation and experiment along the die, it becomes clear that there is a significant pressure drop in areas of cross-sectional change in the experiment, which cannot be represented with pure shear viscosity. With the hybrid viscosity model, however, the flow behavior can be reproduced well in areas of both shear and extension. This enables improved design of WPC extrusion dies using numerical simulations.

In further works, the definition of the extension rate should be changed between uni- or biaxial extension depending on the deformation present. In addition, the hybrid material model can be investigated in more detail on industry-related extrusion dies. Also, the comparison of the flow at different wood contents will provide further insights in the extrusion of WPC.
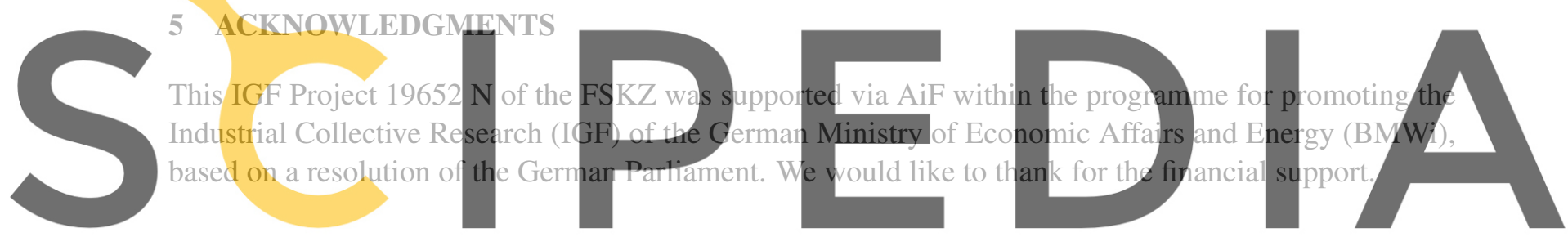

REFERENCES

Register for free at https//www.scipedia.com to download the version without the watermark

[1] Böhme, G., Stromungsmechanik nichtnewtonscher Fluide. Teubner, (2000)

[2] Dammer, L. et al., Current situation and trends of the bio-based industries in Europe with a focus on bio-based materials. Pilot Study for BBI JU. nova-Institue, (2017)

[3] Lebaal, N. et al. Optimisation of extrusion flat die design and die wall temperature distribution, using Kriging and response surface method. International Journal of Materials \& Product Technology (2010) 38:307-322.

[4] Li, T.Q. and Wolcott, M.P. Rheology of HDPE wood composites. I. Steady state shear and extensional flow. Composites Part A: Applied Science and Manufacturing (2004) 35:303-311.

[5] Rosenbaum, E.E. and Hatzikiriakos, S.G. Wall slip in the capillary flow of molten polymers subject to viscous heating. AIChE Journal (1997) 43:598-608.

[6] Sukiman, M.S. et al. Design and numerical modeling of the thermoforming process of a WPC based formwork structure. Materials Today Communications (2020) 22:100805. 
[7] Siegbert, R. et al. Design Criteria in Numerical Design of Profile Extrusion Dies. Key Engineering Materials (2013) 554-557:794-800.

[8] Yatsenko, S. et al., Experimentell abgesicherte Simulationsrechnungen zur beschleunigten Auslegung von WPC-Extrusionswerkzeugen. Shaker, (2020)

[9] Nóbrega, J.M. et al. Flow balancing in extrusion dies for thermoplastic profiles: Part I: Automatic design. International Polymer Processing (2003) 18:298-306.

[10] Carreau, P.J. et al., Rheology of Polymeric Systems: Principles and Applications. Hanser (1996)

[11] Logan, S.R. The origin and status of the Arrhenius equation. Journal of Chemical Education (1982) 59:279-281.

[12] Descher, S., Modeling and Simulation of Crystallization Processes in Polymer Melt Flows. Kassel University Press, (2020)

[13] Tesch, K. On invariants of fluid mechanics tensors. TASK Quarterly (2013) 17:223-230.

[14] Rütten, M., Verallgemeinerte newtonsche Fluide. Springer, (2018)

[15] Ziabicki, A. Crystallization of polymers in variable external conditions. Part II: Effects of cooling in the absence of stress and orientation. Colloid and Polymer Science (1996) 274:705-716.

[16] Xu, B. et al. Viscous dissipatiopn influencing viscosity of polymer melt in micro channels. Journal of Mechanical Science and Technology (2010) 7:1417-1423.
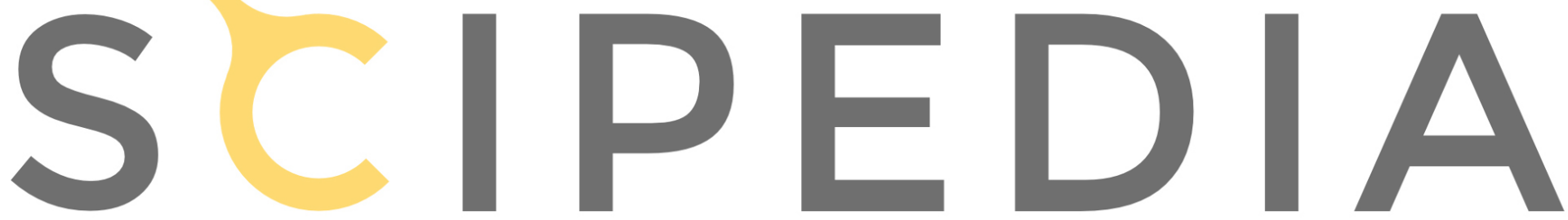

Register for free at https//www.scipedia.com to download the version without the watermark 